\title{
$5-32$

\section{A Technique for Computation of Noise Temperature Due to a Beam Waveguide Shroud}

\author{
W. Veruttipong and M. M. Franco \\ Ground Antennas and Facilities Engineering Section
}

\begin{abstract}
Direct analytical computation of the noise temperature of real beam waveguide (BWG) systems, including all mirrors and the surrounding shroud, is an extremely complex problem and virtually impossible to achieve. Yet the DSN antennas are required to be ultra low-noise in order to be effective, and a reasonably accurate prediction is essential. This article presents a relatively simple technique to compute a real BWG system noise temperature by combining analytical techniques with data from experimental tests. Specific expressions and parameters for $X$-band (8.45-GHz) BWG noise computation are obtained for DSS 13 and DSS 24, now under construction. These expressions are also valid for various conditions of the $B W G$ feed systems, including horn sizes and positions, and mirror sizes, curvatures, and positions. Parameters for $S$ - and Ka-bands (2.3 and $32.0 \mathrm{GHz}$ ) have not been determined; however, those can be obtained following the same procedure as for $X$-band.
\end{abstract}

\section{Introduction}

Noise temperature due to a BWG assembly is one of the major contributers to total antenna receive system noise temperature, especially for an ultra-low-noise system or a system with high spillover power in the BWG shroud. A reasonably accurate prediction of the BWG assembly noise temperature is essential. Direct analytical computation of the noise temperature of real BWG systems, including all mirrors, is an extremely complex problem and virtually impossible to achieve.

This article presents a new technique that combines an analytical approach with data from measurement tests to construct a specific expression with some parameters to compute noise temperature in a real BWG system, including all mirrors. Appendix A gives the needed relationships among reflector material conductivity, resistivity, loss, and resultant noise. Experimental tests that have helped to establish this technique are presented in Appendix B. Appendix $C$ develops the effective noise temperatures, as seen by spillovers in the upper and lower portions of the BWG assembly. Appendix D contains physical explanations of the behavior of the field and noise temperature in the BWG shroud. The results from this technique have been used to predict BWG noise temperatures for DSS-13 Phase II and DSS 24.

\section{Characteristics of the Electromagnetic Fields Inside a BWG Shroud}

The total RF power originating from a horn aperture (viewed in transmission, for convenience) and propagating 
through a BWG shroud $\left(P_{\mathrm{BWG}}\right)$ can be separated into two parts (see Fig. 1)

$$
P_{\mathrm{BWG}}=P_{\mathrm{m}}+P_{\text {spill }}
$$

where $P_{\mathrm{m}}$ is the majority of the total power (usually $>95$ percent of $P_{\mathrm{BWG}}$ ) that is always confined inside all BWG mirrors; it does not contact the BWG wall and there are no multiple reflection, diffraction, or creeping wave components. The power $P_{\mathrm{m}}$ reflects from each and every mirror once before leaving the BWG shroud through the opening near $\mathrm{F}_{1} . P_{\mathrm{m}}$ can be computed easily because BWG wall and mirror interactions are small and ignored, which drastically simplifies the problem. The power that is confined inside the lower mirrors M6 (or M5, M4, etc.) is not considered as $P_{\mathrm{m}}$ because leaks occur along the way up from M6 to M1. For most practical cases where the total spillover loss is less than 5 percent, the total power confined inside the last mirror M1 is approximately used as $P_{\mathrm{m}}$.

$P_{\text {spill }}$ is the sum of spillover powers of each mirror. The $P_{\text {spill }}$ fraction creeps and bounces around the BWG walls, mirrors, brackets (behind the mirrors), and the edges, etc., and suffers dissipation loss and consequent noise. On an average, the $P_{\text {spill }}$ power largely dissipates before a small remainder exits the BWG opening near $F_{1}$ (some power may get back into the horn, but most is dissipated, with consequent transformation into noise). Even though $P_{\text {spill }}$ can be computed accurately $\left(P_{\text {spill }}=P_{\mathrm{BWG}}-P_{\mathrm{m}}\right)$, its field distribution and its chaotic behavior inside the lossy BWG shroud is virtually impossible to compute analytically.

\section{Noise Temperature Contributions Inside the BWG Shroud}

From Eq. (1), the corresponding noise temperatures are

$$
T_{\mathrm{BWG}}=T_{\mathrm{m}}+T_{\mathrm{spill}}
$$

where $T_{\mathrm{BWG}}$ is the total noise temperature (in kelvins) due to the BWG (including the shroud, mirrors, brackets, etc.). The values $T_{\mathrm{m}}$ and $T_{\text {spill }}$ are the noise temperature contributions from $P_{\mathrm{m}}$ and $P_{\text {spill }}$, respectively. Because of the simplicity of $P_{\mathrm{m}}$, its corresponding noise temperature $T_{\mathrm{m}}$ can be computed with acceptable accuracy. The details are given in Appendix A. From Appendix A [Eq. (A-6)], the noise temperature $T_{\mathrm{m}}$ for DSS 13 or DSS 24 in kelvins at X-band is

$$
T_{\mathrm{m}}=0.734 P_{\mathrm{m}} / P_{\mathrm{BWG}}=0.734 \alpha_{\mathrm{m}}
$$

where $\alpha_{\mathrm{m}}$ is the $P_{\mathrm{m}}$ fraction of $P_{\mathrm{BWG}}$, dimensionless.

Similarly, because of the complexity of the field that contributes to $P_{\text {spill }}$, it is not possible to analytically compute $T_{\text {spill }}$. Unfortunately, $T_{\text {spill }}$ is usually a major contribution to $T_{\mathrm{BWG}}$ (for example, DSS-13 Phase II, X-band $T_{\text {spill }} \sim 3.0 \mathrm{~K}$ and $T_{\mathrm{m}} \sim 0.7 \mathrm{~K}$ ) and a reasonably accurate prediction is essential. The following section shows a technique used to compute $T_{\text {spill. }}$.

\section{A Technique for $\boldsymbol{T}_{\text {spill }}$ Computation}

Noise temperature due to spillover power $P_{\text {spill }}$ is given in a very simple form as

$$
T_{\mathrm{spill}}=\left(P_{1} / P_{\mathrm{BWG}}\right) T_{1}+\left(P_{2} / P_{\mathrm{BWG}}\right) T_{2}=\alpha_{1} T_{1}+\alpha_{2} T_{2}
$$

where $P_{1}$ is the total spillover power of the two mirrors (M5 and M6) in the basement and the value $P_{2}$ is the total spillover power of the four mirrors (M1, M2, M3, and M4) above the basement ceiling. The values $\alpha_{1}$ and $\alpha_{2}$ are the normalized powers (with respect to $P_{\mathrm{BWG}}$ ) of $P_{1}$ and $P_{2}$, respectively. The values $T_{1}$ and $T_{2}$ are source (or sink) effective noise temperatures seen by $P_{1}$ and $P_{2}$, in kelvins, respectively. In other words, $T_{1}$ and $T_{2}$ are effective noise temperatures that $\alpha_{1}$ and $\alpha_{2}$ see in the BWG assembly (including the shroud, mirrors, brackets, etc.). For example, if the BWG shroud were very short and highly conducting, $T_{1}$ and $T_{2}$ would be equal and approximately $T_{\text {sky }}$. If the shroud were very long and lossy, $T_{1}$ and $T_{2}$ would again be equal and approximately $273.16+T_{\text {celsius }}(\sim 290 \mathrm{~K})$.

If one substitutes Eqs. (3) and (4) into Eq. (2), the BWG shroud noise temperature at X-band becomes

$$
T_{\mathrm{BWG}}=0.734 \alpha_{\mathrm{m}}+\alpha_{1} T_{1}+\alpha_{2} T_{2}
$$

It is noted that $\alpha_{\mathrm{m}}+\alpha_{1}+\alpha_{2}=1.0$.

The value $T_{\mathrm{BwG}}$ was measured at DSS 13 . The power fractions $\alpha_{\mathrm{m}}, \alpha_{1}$, and $\alpha_{2}$ in Eq. (5) are known and the unknown quantities are $T_{1}$ and $T_{2}$. By performing various measurements and perturbations at X-band, the coefficients $T_{1}$ and $T_{2}$ at X-band have been obtained (see details in Appendices B and C).

DSS 13, no basement shroud: 


$$
\begin{aligned}
& T_{1}=300 \pm 10 \mathrm{~K} \\
& T_{2}=240 \pm 45 \mathrm{~K}
\end{aligned}
$$

DSS 24, full shroud:

$$
\begin{aligned}
& T_{1}=280 \pm 20 \mathrm{~K} \\
& T_{2}=230 \pm 45 \mathrm{~K}
\end{aligned}
$$

It is noted that Eqs. (6a) and (6b) both have steel shrouds (the same conductivity as DSS 13) above the basement céiling, while DSS 24 has an additional large aluminum shroud in the basement.

In general, the coefficients $\left(T_{1}, T_{2}\right)$ apply to any $\mathrm{BWG}$ antenna with a similar shroud structure and (nearly) the same conductivity. The same coefficients are valid for various conditions in a BWG system, including the horn sizes and positions, and mirror sizes, curvatures, and positions. See Appendix D for more physical explanations about the behavior of field and noise temperature due to the BWG assembly.
The effective temperatures $T_{1}$ and $T_{2}$ for S- and Kabands are expected to be slightly smaller and larger than the $\mathrm{X}$-band coefficients, respectively. The accurate values of the S- and Ka-bands coefficients have not been determined.

\section{Conclusion}

A technique for computing a BWG assembly noise temperature has been established and the technique can now be applied to the low-noise DSN BWG antennas. Specific expressions and parameters for X-band BWG noise temperature computation of DSS 13 and DSS 24 are obtained from Eqs. (5) and (6). These expressions are valid for various conditions of the BWG feed system, including the horn sizes and positions, and the mirror sizes, curvatures, and positions. The coefficients in Eq. (6) are not sensitive to small variations of BWG shroud structure (diameter, length, etc.), shroud conductivity, or operating frequency. Parameters for S- and Ka-bands have not been determined; however, they will not be dramatically different and can be obtained by following the same procedure as for X-band. The BWG noise temperature computed by this technique should be, at best, as accurate as a measurement result (plus some small error for the $P_{\text {spill }}$ computation) and should be valid for all engineering applications.

\section{Acknowledgments}

The authors wish to thank T. Y. Otoshi for initiating an experimental test of DSS 13. The test enabled a better understanding of the problems of noise temperature due to a BWG assembly and provided valuable data used in this article. Thanks also to D. A. Bathker for his help with Appendix A and to the personnel of DSS 13 for their support. 


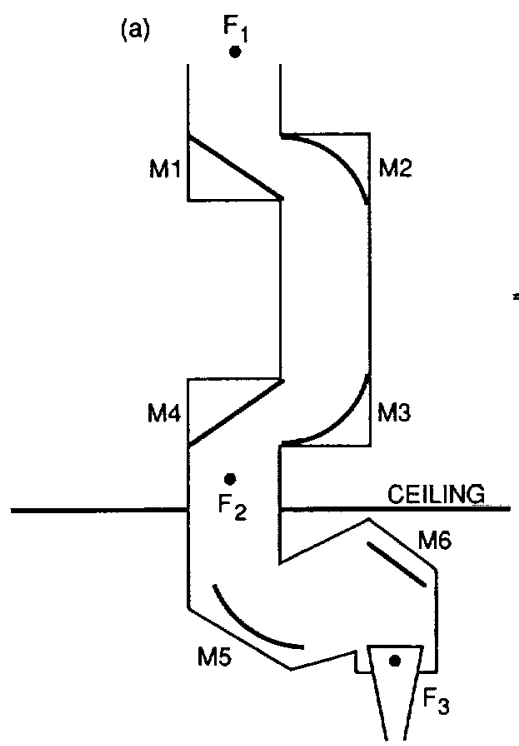

(b)

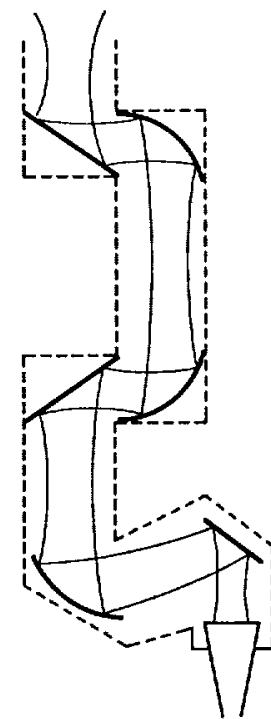

(c)
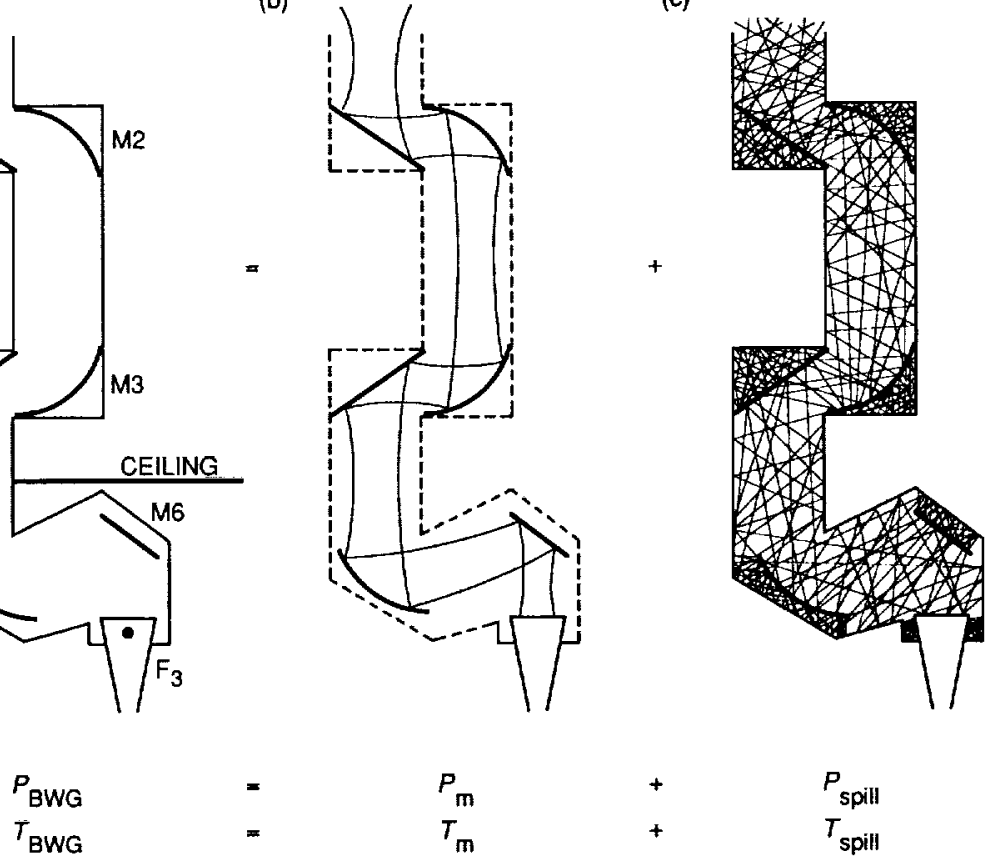

$\begin{array}{lll}P_{\mathrm{m}} & + & P_{\text {spill }} \\ T_{\mathrm{m}} & + & T_{\text {spill }}\end{array}$

Fig. 1. Characteristics of the fields inside a BWG shroud and thelr corresponding nolse temperatures. 


\section{Appendix A}

\section{Noise Temperature Due to BWG Mirror Surface Resistivity}

Noise temperature due to one reflection of a wave normally incident on a mirror surface is ${ }^{1}$

$$
\begin{gathered}
T \sim \frac{4 R_{s}}{Z_{o}} T_{p} \alpha, \text { kelvins } \\
R_{s}=0.02 \pi \sqrt{\frac{f}{10 \sigma_{N}}}, \quad \text { ohms/square }
\end{gathered}
$$

where

$$
\begin{aligned}
R_{s} & =\text { surface resistivity of the mirror, ohms/square } \\
f & =\text { frequency, GHz } \\
\sigma_{N} & =\text { normalized conductivity }=\sigma \times 10^{-7}, \text { mho/meter } \\
Z_{o} & =\text { free-space wave impedance, ohms } \\
T_{p} & =\text { physical temperature, kelvins } \\
\alpha & =\text { power fraction illuminating the mirror }
\end{aligned}
$$

For a circularly polarized wave with an average incident angle to the mirror equal to $\theta$, an approximate expression for $T$, valid where $\theta$ is away from grazing incidence, is

$$
T \sim \frac{4 R_{s}}{Z_{o}} T_{p} \alpha \frac{1}{2}\left(\cos \theta+\frac{1}{\cos \theta}\right)
$$

The total noise temperature of $n$ mirrors is

$$
T=\frac{2 T_{p}}{Z_{o}} \sum_{i=1}^{n} R_{s i} \alpha_{i}\left(\cos \theta_{i}+\frac{1}{\cos \theta_{i}}\right)
$$

where $R_{s i}, \alpha_{i}$, and $\theta_{i}$ are $R_{s}, \alpha$, and $\theta$ of the $i$ th mirror, respectively.

\footnotetext{
1 D. A. Bathker, "Planewave Reflection Noise Temperature Due to Surface Resistivity," JPL Interoffice Memorandum 3328-92-0144 (internal document), Jet Propulsion Laboratory, Pasadena, California, August 20, 1992.
}

In order to compute $T_{m}$ [as shown in Eq. (2) for DSS 13 or DSS 24], the noise temperature in Eq. (A-4) can be used with the following conditions:

(1) The surface conductivity is the same for all six mirrors with $\sigma=2.3 \times 10^{7} \mathrm{mho} / \mathrm{meter}$ (for 6061T6 aluminum).

(2) The power fraction is also the same for all mirrors and equal to $\alpha_{\mathrm{m}}$, where $\alpha_{\mathrm{m}}$ is the main fraction of $P_{\mathrm{BWG}}\left(\alpha_{\mathrm{m}}=P_{\mathrm{m}} / P_{\mathrm{BWG}}\right)$.

(3) The incident angle is $\theta=45 \mathrm{deg}$ for the four mirrors (M1, M2, M3, and M4) above the ceiling and $\theta=30$ deg for the two basement mirrors (M5 and M6).

(4) The physical temperature $T_{p}=290 \mathrm{~K}$.

(5) The frequency $f=8.45 \mathrm{GHz}$ will be used because all measured data in Appendix $\mathrm{C}$ are at $8.45 \mathrm{GHz}$.

From Eq. (A-4) and the above five conditions, the total noise temperature due to surface resistivity of all six mirrors of DSS 13 or DSS 24 becomes

$$
\begin{aligned}
T_{\mathrm{m}}= & \frac{2 T_{p} R_{s} \alpha_{\mathrm{m}}}{Z_{o}}\left\{2\left(\cos 30+\frac{1}{\cos 30}\right)\right. \\
& \left.+4\left(\cos 45+\frac{1}{\cos 45}\right)\right\}
\end{aligned}
$$

Substitute $T_{p}=290, Z_{o}=120 \pi, f=8.45$, and $\sigma_{N}=2.3$ into Eqs. (A-2) and (A-5), and one obtains

$$
T_{\mathrm{m}}=0.734 \alpha_{\mathrm{m}}
$$

Note that in a reasonable design with $\alpha_{\mathrm{m}}=0.95$ or more, the dissipation of six aluminum BWG mirrors alone causes a noise component of about 0.7 kelvin at $\mathrm{X}$-band. 


\section{Appendix B}

\section{BWG Noise Temperature Experimental Tests}

T. Y. Otoshi and M. M. Franco performed several measurements at DSS 13 in order to gain a better understanding of the BWG assembly noise temperature. The results show a higher than (initially) expected noise temperature (which was about $4 \mathrm{~K}$ ). By adding skirts around the rim of the BWG mirrors in these tests, the noise temperature was reduced only a fraction of a kelvin. The two mirrors in the basement normally do not have shrouds. After constructing a temporary shroud (made with lightweight insulating material, aluminum foil-coated on two sides, and a foam center) that partially enclosed the two mirrors, the noise temperature dropped only $0.25 \mathrm{~K}$.

The above results led to further investigation by W. Veruttipong and M. M. Franco. The experimental tests described below (performed by Veruttipong and Franco between May 31 and June 3,1991) help to explain why the BWG noise temperature is higher than initially expected. More important, their results led to a new technique used to compute the BWG noise temperature.

Experiment 1. The purpose of this experiment was to observe the effect of the basement shroud on system noise temperature.

A tightly fitted and fully enclosed basement shroud was made from a partially enclosed shroud left over from Otoshi and Franco's experiment (see Fig. B-1). The same foil material was used to patch all the opening spaces. Aluminum tape was used to seal all small gaps. J. Withington built an RF probe to check for any RF signal leaks around the new shroud. The JPL standard 22-dB X-band corrugated horn was used, and its aperture was positioned at 3.34 in. above $F_{3}$ throughout the experiments. The following system noise temperatures were recorded:

Fully enclosed basement shroud

$$
T_{\mathrm{op}}=33.62 \mathrm{~K}
$$

Partially enclosed basement shroud $\quad\rangle \Delta=0.23 \mathrm{~K}$

$$
T_{\mathrm{op}}=33.85 \mathrm{~K}
$$

No basement shroud

$$
\rangle_{\Delta}=0.25 \mathrm{~K}
$$

$$
T_{\mathrm{op}}=34.10 \mathrm{~K}
$$

Conclusion 1 . The results clearly show that the basement shroud does not significantly reduce the BWG noise temperature. In other words, the spillover field in the basement still sees a high effective noise temperature (close to $300 \mathrm{~K}$ ) with or without the basement shroud.

Experiment 2. The purpose of this experiment was to reconfirm the results from Experiment 1 that spillover power sees a high effective noise temperature in the BWG shroud.

The main field inside the BWG shroud $\left(P_{\mathrm{m}}\right)$ was excited so that it had a characteristic similar to $P_{\text {spil }}$ in Fig. 1. By causing mirrors M5 and M6 to be rippled (by putting curly aluminum tape all over the mirror surfaces), the field inside the shroud was obviously chaotic and similar to Fig. E-1(c). The tightly fitted basement shroud was still in place and the noise temperature reading was $235.5 \mathrm{~K}$.

Conclusion 2. The simulated scattered field is not quickly guided by the shroud to the sky. In fact, it bounces around and effectively creates a high dissipation loss. The $235.5 \mathrm{~K}$ does not represent any specific parameter. It is just an indicator showing that the simulated scattered field (or spillover field in the real BWG) sees an effective noise temperature on the order of $200 \mathrm{~K}$. The accurate value is computed in Appendix C. Earlier in the experiment, it was not fully understood that the effective noise temperature for spilled or scattered power was approximately $T_{\text {sky }}$, and that is why the measured noise temperature was higher than expected.

Experiment 3. The purpose of this test was to see whether there would be any unusual noise temperature measurements when the shroud was short-circuited at various locations. The intent was to see whether the noise temperature readings were high (300 $\mathrm{K} \pm \Delta)$, medium $(150 \mathrm{~K}$ $\pm \Delta$ ), or low ( $50 \mathrm{~K} \pm \Delta$ ). Therefore, a very high-precision experimental setup was not necessary.

The short circuit was done by closing the BWG tube with a foam-backed foil disk. Five of the six mirror surfaces were intentionally rippled (see Experiment 2) and the noise temperature readings were

$$
\begin{aligned}
& \text { Short circuit near } \mathrm{F}_{1}: T_{\mathrm{op}, 1}=296 \mathrm{~K} \\
& \text { Short circuit at the ceiling: } T_{\mathrm{op}, 2}=304 \mathrm{~K}
\end{aligned}
$$


Short circuit just above the cement: $T_{\mathrm{op}, 3}=304 \mathrm{~K}$ Short circuit near $F_{1}$ (all mirror surfaces are good): $T_{\mathrm{op}, 4}=131 \mathrm{~K}$

Conclusion 3. All readings (except $T_{\mathrm{op}, 4}$ ) are $300 \mathrm{~K}$ $\pm 5 \mathrm{~K}$, as expected, because a perfectly closed cavity has an ambient noise temperature. The slightly lower reading of $T_{\mathrm{op}, 1}$ results from an imperfect short circuit near $F_{1}$ due to a relatively strong wind. It is noted that if all the mirror surfaces are good during the measurement, the noise temperatures will differ greatly, depending upon the location of the short circuit. For example, if the short circuit is exactly at the waist of the beam (near $F_{1}$ or $\mathrm{F}_{2}$ ), the noise temperature will be low (less than $100 \mathrm{~K}$ ) because most of the energy will get back to the horn. The noise temperature $T_{\mathrm{op}, 4}$ is not less than $100 \mathrm{~K}$ because the short-circuit location is not at the waist of the field and the short-circuit disk is neither perfectly level nor flat.
Experiment 4. The purpose of this test was to measure the effect of a section of concrete in the shroud (see the location of the concrete in Fig. B-1).

To investigate the impact of a concrete surface segment (unlined shroud portion) on the BWG noise temperature, the BWG system was returned to its normal operational condition, i.e., no rippled surfaces, no short circuit, and no basement shroud. One-inch foam-backed foil was used to cover the concrete surface. System noise temperatures were recorded with and without the covering. The result shows practically no change in the noise temperature.

Conclusion 4. The concrete in the BWG shroud is not a major source of BWG shroud noise and the concrete does not create extra noise temperature in the BWG system. This was expected, as the concrete section is near a beam waist.

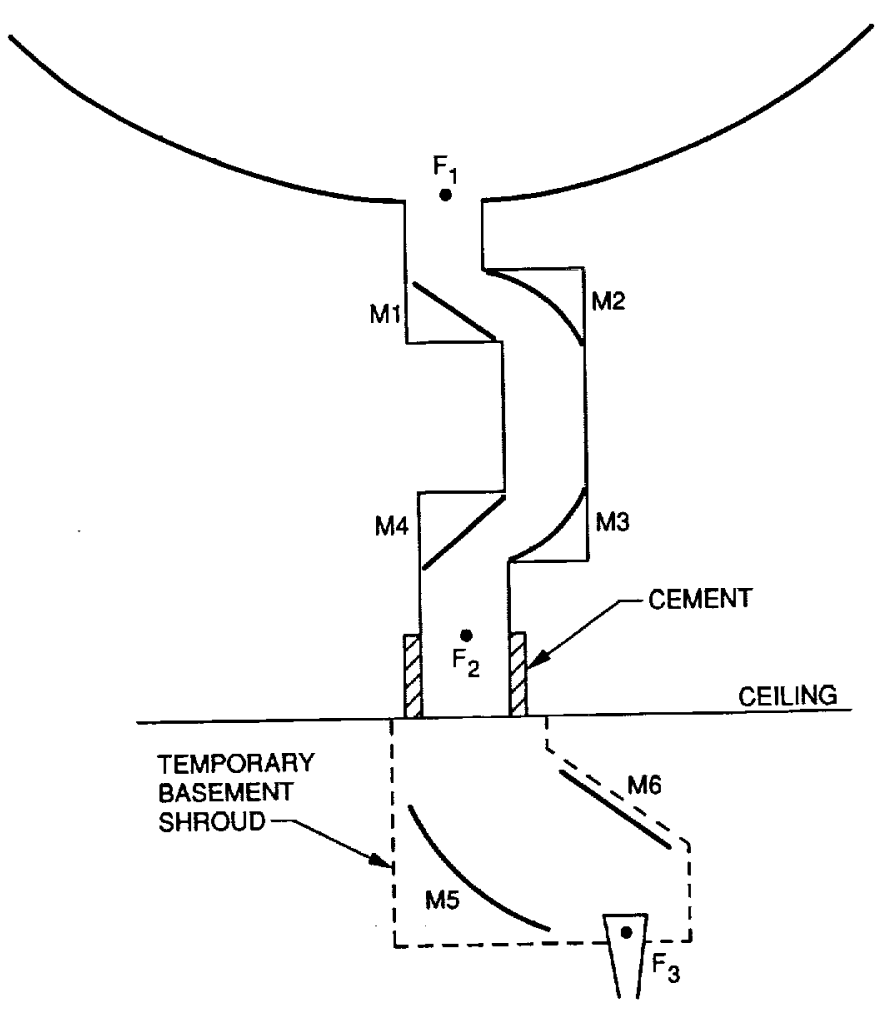

Fig. B-1. DSs-13 BWG antenna with a temporary basement shroud. 


\section{Appendix C}

\section{Effective Noise Temperatures $\left(T_{1}\right.$ and $\left.T_{2}\right)$}

The noise temperature coefficients or effective noise temperatures, as shown in Eq. (4), will be evaluated at X-band for DSS 13 and DSS 24. Recall Eq. (5),

$$
T_{\mathrm{BWG}}=0.734 \alpha_{\mathrm{m}}+\alpha_{1} T_{1}+\alpha_{2} T_{2}
$$

The coefficients for DSS 13 will be considered first. The measurement at DSS 13 reveals $T_{\mathrm{BWG}}=8.9 \pm 0.4 \mathrm{~K}$ [1]. The spillover power of every mirror for DSS 13 is computed, without the BWG wall, by using Physical Optics (PO) software, and the power is normalized to the total horn output power. The results are

$$
\begin{aligned}
\alpha_{\mathrm{m}} & =0.9694 \\
\alpha_{1} & =0.0138 \\
\alpha_{2} & =0.0168
\end{aligned}
$$

Since there is no shroud in the basement of DSS $13, \alpha_{1}$ is mostly dissipated in the basement, therefore,

$$
T_{1}=300 \mathrm{~K}
$$

Substitute $T_{\mathrm{BWG}}=8.9 \mathrm{~K}$, Eqs. (C-1) and (C-2) into Eq. (5), and one obtains

$$
T_{2} \sim 240 \mathrm{~K}
$$

For the deviation of $T_{\mathrm{BWG}}$ of $\pm 0.4 \mathrm{~K}$ and the spillover power within \pm 0.0005 for $\alpha_{1}$ and \pm 0.0010 for $\alpha_{2}$, the deviation of $T_{2}$ becomes $\pm 45 \mathrm{~K}$. It is noted that the value of $T_{1}=300 \mathrm{~K}$ is quite well known and is an accurate value (a deviation of $\pm 10 \mathrm{~K}$ may be applicable). Therefore, the accuracy of $T_{2}$ is nearly as good as the measurement can get (plus a small error in PO computation).
Noise temperature coefficients for DSS 24 will be considered next. From Experiment 1 in Appendix B, the difference in noise temperatures of DSS 13 with and without a basement shroud is about $0.5 \mathrm{~K}$. DSS 24 has a larger basement shroud than the one in the test (Fig. B-1), which results in a slightly higher system noise temperature (about 0.1 to $0.3 \mathrm{~K}$ higher). However, the smaller gap between mirrors and the BWG wall of DSS 24 helps to reduce the noise temperature by about $0.2 \mathrm{~K}$. On balance, it is reasonable to say that the result from DSS 13 with the shroud should approximately represent DSS 24 . Recall that with the temporary basement shroud, the DSS-13 noise temperature decreased by $0.5 \mathrm{~K}$. One can assume that $0.3 \mathrm{~K}$ is related to $\alpha_{1}$ and $0.2 \mathrm{~K}$ is related to $\alpha_{2}$ (gap effect included). Even though this assumption of the ratio contribution may be questionable, the total noise temperature difference of $0.5 \mathrm{~K}$ is always preserved.

From Eqs. (5) and (C-1), one can write

$$
\begin{aligned}
& \Delta T_{1}=\frac{0.3}{\alpha_{1}}=\frac{0.3}{0.0138} \sim 20 \mathrm{~K} \\
& \Delta T_{2}=\frac{0.2}{\alpha_{2}}=\frac{0.2}{0.0168} \sim 10 \mathrm{~K}
\end{aligned}
$$

From Eqs. (C-2), (C-3), and (C-4), the coefficients for DSS 24 are

$$
\begin{aligned}
& T_{1}=300-20=280 \mathrm{~K} \\
& T_{2}=240-10=230 \mathrm{~K}
\end{aligned}
$$

The deviation of $T_{1}$ for DSS 24 should be increased to $\pm 20 \mathrm{~K}$ due to a small uncertainty $(\sim \pm 0.1 \mathrm{~K})$ of 0.5 - $\mathrm{K}$ power distribution. The deviation of $T_{2}$ for DSS 24 should be approximately the same as DSS 13 , which is about $\pm 45 \mathrm{~K}$.

\section{Reference}

[1] T. Y. Otoshi, S. R. Stewart, and M. M. Franco, "Portable Microwave Test Packages for BWG Antenna Performance Evaluations," IEEE Trans. Microwave Theory Tech., vol. 40, no. 6, June 1992. 


\section{Appendix D}

\section{Field and Noise Temperature Due to a BWG Shroud}

The spillover power past a mirror is defined here as a power originating from a single source that is not incident on the mirror in the absence of a BWG wall and other mirrors. In reality, with BWG walls and all mirrors included, the spillover power of each mirror will spread (bounce arqund) nonuniformly throughout the shroud. The dissipation losses are associated with the conductivities of the walls, mirrors, brackets, etc., in a very complex and undetermined combination. Total spillover noise temperature is the sum of the contributions of all the mirrors. Besides the complexities of the scattered fields related to the mirror brackets, edges, bolts and nuts, welding scars, gaps between mirrors and BWG walls, etc., one must also deal with a partially open cavity problem of the lossy BWG shroud. Based on over five years' experience in fundamental research with reflection, diffraction, and creeping waves of various surfaces and objects, it is concluded that the characteristic of $P_{\text {spill }}$ (and $T_{\text {spill }}$ ) is so chaotic in a very lossy and complex system that it is not possible to compute analytically.

One can draw a similarity between the expression $T_{\text {spill }}=\alpha_{1} T_{1}+\alpha_{2} T_{2}$ in Eq. (4) and Ohm's law $V=I R$. The resistance $R$ (in ohms) is a result of thermal agitation of electrons, which prevents current (free electrons) from moving freely through the resistor $R$. The relation $V=I R$ applies for a macroscopic region in an electric circuit, and $R$ is obtained from a relation $R=V / I$. It is extremely difficult to compute $R$ from a microscopic point of view by computing the total energy loss from continual collisions of electrons or by determining an average rate of flow of free electrons through the thermally excited lattice structure of the resistor. The resistance $R$ is linear and isotropic within a wide range of $I$ (similary, $T_{1}$ and $T_{2}$ are linear and perhaps isotropic). The linearity of $T_{1}$ (similarly for $T_{2}$ ) in Eq. (4) physically means that the behavior (or the degree of chaos) of $\alpha_{1}$ is approximately the same, regardless of the magnitude of $\alpha_{1}$ (as long as there is no dielectric breakdown).

The reason why $T_{\text {spill }}$ in Eq. (4) is separated into two parts (above and below the subterranean room ceiling) is that the shroud in the basement is very different from the shroud above. In fact, DSS 13 does not have a basement shroud at all. Fortunately, this helps to improve the accuracy of Eq. (6a) because $T_{1}=300 \mathrm{~K}$ is an accurate effective noise temperature for a very large basement room, provided that the exit "hole" is a small area, as compared with the total basement surface area. This leads to an accurate determination of $T_{2}$ because it is the only remaining unknown. Adding small perturbations with the guidance from measurement results leads to $T_{1}$ and $T_{2}$ of DSS 24, as shown in Eq. (6b). Suppose that a horn were located at $\mathrm{F}_{2}$ and there were no basement. The coefficient $T_{1}$ does not exist, $T_{2}$ will be smaller than the one given in Eq. (6b) because there is no (close to) room temperature load below $\mathrm{F}_{2}$.

For low spillover loss in a BWG shroud, the total antenna system noise temperature is not sensitive to a small variation (say, $\pm 20 \mathrm{~K}$ ) of $T_{1}$ or $T_{2}$. For example, for DSS-13 Phase II X-band with a total system noise temperature of $35 \mathrm{~K}$ and normalized spillover power $\alpha_{\text {spill }}=0.012, \pm 20 \mathrm{~K}$ variations in $T$ resulted in only $0.012( \pm 20)= \pm 0.24 \mathrm{~K}$. Also, for an extremely low spillover loss (one mirror spill $<0.05$ percent), the noise temperature computation may not give a sensible result because of the possibility of inaccurate spillover computation. 